\title{
Association of Tetanus-Diphtheria Vaccination during Pregnancy with Preeclampsia
}

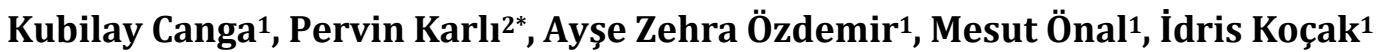 \\ ${ }^{1}$ Department of Obstetrics and Gynecology, Ondokuz Mayıs University Research Hospital, Samsun, Turkey \\ ${ }^{2}$ Department of Obstetrics and Gynecology, Amasya University Research Hospital, Amasya, Turkey \\ Email: *parpi2300@hotmail.com
}

How to cite this paper: Canga, K., Karll, P., Özdemir, A.Z., Önal, M. and Koçak, İ. (2018) Association of Tetanus-Diphtheria Vaccination during Pregnancy with Preeclampsia. Open Journal of Obstetrics and Gynecology, 8, 1130-1139.

https://doi.org/10.4236/ojog.2018.812114

Received: September 8, 2018

Accepted: October 9, 2018

Published: October 12, 2018

Copyright $\odot 2018$ by authors and Scientific Research Publishing Inc. This work is licensed under the Creative Commons Attribution International License (CC BY 4.0).

http://creativecommons.org/licenses/by/4.0/

\begin{abstract}
Introduction: The tetanus-diphtheria vaccine administered during pregnancy is used as a toxoid vaccine with an intact antigen structure. In this study, we investigated the association of tetanus-diphtheria vaccination (TDV) during pregnancy with adverse maternal and neonatal outcomes and particularly preeclampsia that may occur during pregnancy. Materials and Methods: From the patients who presented to our clinic between 01.01.2016 and 31.12.2016 and underwent a cesarean section, a total of 435 patients, 327 who received TDV and 108 who did not receive TDV, were included in the study. The groups were compared with respect to their demographic characteristics. The patient data on the gestational week, birth weight, normal newborn, preeclampsia, small for gestational age (SGA), early membrane rupture, preterm labor, diabetic mother's baby, prematurity, temporary neonatal tachypnea, newborn's need for intensive care, and neonatal mortality were recorded. Results: While $14.7 \%$ of the women who had been administered TDV had preeclampsia diagnosis during their pregnancy, $12 \%$ of those who had not been vaccinated were diagnosed with preeclampsia. This showed no significant correlation between having been vaccinated during pregnancy and preeclampsia $(\mathrm{p}=0.474)$. The other outcomes we had specified had also no statistically significant correlations with vaccination during pregnancy. Having been vaccinated during pregnancy was found correlated only with the place of residence and nulliparity ( $\mathrm{p}=0.004$ and $\mathrm{p}=0.002$, respectively). Conclusion: No correlation was found in the study between TDV during pregnancy and preeclampsia. According to the data obtained from this study, vaccination was found to have no negative effect on preeclampsia or the other outcomes.
\end{abstract}

\section{Keywords}

Preeclampsia, Tetanus-Diphtheria Vaccination, Vaccination in Pregnancy, 
Vaccination, Pregnancy

\section{Introduction}

Preeclampsia is defined as a new onset of hypertension and proteinuria or hypertension and target organ dysfunction with/without proteinuria after the $20^{\text {th }}$ gestational week in a previously normotensive pregnant woman [1]. It has been realized today that proteinuria does not necessarily accompany preeclampsia in some pregnant women with this disease [2]. For this reason, the American College of Obstetricians and Gynecologists (ACOG) proposed in 2013 some other diagnostic criteria mentioned below, but proteinuria remains to be a major diagnostic criterion [3]. Being an anaerobic gram-positive bacterium, Clostridium tetani thrives in dirt, dust and digestive systems of some animals. Tetanus is caused by a neurotoxin originating from $C$. tetani in contaminated wounds [4]. Neonatal tetanus kills approximately 500,000 infants every year. Additionally, 15,000 - 30,000 women worldwide are reported to die due to tetanus annually. The cause of this disease is the development of wound infection due to after delivery, miscarriage or surgery. Approximately 50 incidences of tetanus are reported in the USA every year and most of these involve those over 60 years of age, but some incidences can be seen also in children and newborns [5]. Routinely practiced in many countries as a vaccination scheme during pregnancy to prevent neonatal tetanus, which is so important in protecting public health, of tetanus-diphtheria vaccination (TDV) is also being questioned with no clear-cut answer for constituting a risk factor for preeclampsia by contributing the pathogenesis of other diseases during pregnancy while it prevents neonatal tetanus, which has a high level of infective morbidity and mortality. With this study, it was planned to assess whether or not TDV has any impact on maternal and neonatal outcomes and particularly on the probability of developing preeclampsia.

\section{Materials and Methods}

In this study, the Ondokuz Mayıs University School of Medicine, Samsun, Turkey's regional data are used. From the patients who presented to our clinic between 01.01.2016 and 31.12.2016 and underwent a cesarean section, a total of 435 patients, 327 who received TDV and 108 who did not receive TDV, were included in the study. We have conducted this study using one-year patient data. We haven't calculated minimum sample size. The patient data on the gestational week, birth weight, normal newborn, presence of preeclampsia, SGA, early membrane rupture, preterm labor, diabetic mother's baby, prematurity, temporary neonatal tachypnea, newborn's need for intensive care and neonatal mortality were recorded. From the file records of the patients who were diagnosed with preeclampsia, their diagnoses were checked in line with the ACOG 2013 rec- 
ommendations if they met the preeclampsia diagnosis criteria (systolic blood pressure being $\geq 140 \mathrm{mmHg}$ and/or diastolic blood pressure being $\geq 90 \mathrm{mmHg}$ in two measurements taken at a 4 -hour interval after the $20^{\text {th }}$ gestational week of a pregnant woman who had been normotensive previously and her proteinuria being $\geq 0.3 \mathrm{~g}$ in a 24 -hour urine or her protein/creatinin rate being $\geq 0.3 \mathrm{mg} / \mathrm{mg}$ or $30 \mathrm{mg} / \mathrm{mmol}$ ). The vaccinated and unvaccinated groups were compared with respect to age, place of residence, body mass index, nulliparity, and smoking status. Women who had chronic hypertension, heart disease, type 1 or type 2 diabetes, kidney disease, a known disease that would affect liver enzyme levels, autoimmune disease or placenta anomaly as well as those who had another type of vaccination during their pregnancy, who did not have a vaccination calendar from which we could confirm whether or not they had TDV during their pregnancy, who had a pregnancy involving fetal congenital malformation, those whose pregnancy was earlier than the $24^{\text {th }}$ gestational week, and those who had intrauterine death or multiple pregnancy were excluded from the study. Every patient was phoned to inform them about the study and to ask if they were willing to take part in the study. Those who volunteered were asked first if they had TDV during their pregnancy, and if they did, when and how many doses of it they had. The women answered by checking their vaccination statuses from their current vaccine tracking cards. Those who did not remember whether or not they had the vaccination were excluded from the study. The patients were then asked if they had TDV before their pregnancy for some reason. However, since a large percentage of them did not remember it, our study was designed without taking prior vaccinations into consideration. Subsequently, information on their residences, prenatal weights, heights, pregnancy treatments, known diseases and smoking statuses and the drugs they used during their pregnancy was obtained. The diagnostic information on the mother and newborn, patient anamneses, operation reports, and daily newborn intensive care epic rises were obtained from the medical information system of our hospital. The IBM SPSS (Statistical Package for Social Sciences) V23 was used for statistical analyses. The data were checked for normal distribution using the Kolmogorov Smirnov test. The Kruskal Wallis and Mann Whitney $U$ tests were used to comparing the quantitative data not showing normal distribution. The qualitative data were analyzed using the chi-square test. The quantitative data were presented in medians (minimum-maximum) and the qualitative data in frequencies (percentages). The significance level was taken as $\mathrm{p}<0.05$.

Ethical approval: This article was approved by the Ethics Committee of the Faculty of Medicine of Ondokuz Mayıs University, Decision No 2017/24. Informed consent was obtained in writing from all participants.

\section{Results}

From the 435 patients in the study, the number of those who had vaccination during their pregnancy was 327 and that of those who did not have vaccination 
was 108. The median value of age did not differ between the groups ( $p=0.751)$. The mean age of those who had vaccination during their pregnancy was $29.53 \pm$ 6.030 and that of those who did not have vaccination was $29.73 \pm 6.049$. There was no statistically significant difference between the groups in terms of body mass index $(B M I)(p=0.475)$. The mean BMI was $25.65 \pm 5.185$ in those who were vaccinated and $26.14 \pm 6.054$ in those unvaccinated. Similarly, when the body mass index was categorized as $<25,25-30$ and $>30$, there was no association between them with respect to vaccination status ( $\mathrm{p}=0.338)$.

The place of residence was an urban area in $46.4 \%$ of the vaccinated pregnant women and in $30.5 \%$ of the unvaccinated pregnant women. Vaccination during pregnancy was found associated with place of residence $(\mathrm{p}=0.004)$. While $82.2 \%$ of those living in an urban area had the vaccination, only $70 \%$ of those living in a rural area were vaccinated. The rate of vaccination was found lower in rural areas. Nulliparae constituted $49.3 \%$ of those who were vaccinated and $32.5 \%$ of those unvaccinated. A statistically significant correlation was found between nulliparity and vaccination $(\mathrm{p}=0.002$ ). The vaccination rate was $69.5 \%$ in multiparae and $82.1 \%$ in nulliparae. The rate of smokers was $11 \%$ in vaccinated pregnant women and $12 \%$ in unvaccinated ones. While $73.5 \%$ of smokers had the vaccination, the rate was $75.4 \%$ in nonsmokers. Vaccination was not correlated with smoking ( $\mathrm{p}=0.907)$. The birth week was also not correlated with vaccination ( $\mathrm{p}=0.992)$. The median birth week was 38 and the mean birth week $36.99 \pm 2.856$ in those who were vaccinated and 37.6 and $36.97 \pm 2.866$ respectively in those unvaccinated. The median birth weight also did not differ with respect to vaccination status $(\mathrm{p}=0.995)$. The median value was 3070 grams and the mean value $2899.69 \pm 822.172$ grams in those who were vaccinated and 3067.5 grams and $2921.76 \pm 758.829$ grams respectively in those unvaccinated (Table 1).

Of the pregnant women who were vaccinated during their pregnancy, $57.8 \%$ gave full-term birth and had no neonatal health problems. This rate was $59.3 \%$ in those unvaccinated $(\mathrm{p}=0.789)$. Preeclampsia occurred in $14.7 \%$ of the vaccinated women and in $12 \%$ of unvaccinated women during their pregnancy. Therefore, there was no statistically significant correlation between vaccination during pregnancy and preeclampsia $(\mathrm{p}=0.474)$. There was also no statistically significant correlation between vaccination during pregnancy and prematurity, SGA, diabetic mother's baby, preterm labor (\%3.1) early membrane rupture (\%2.8) or temporary neonatal tachypnea. Receiving intensive care service was not correlated with vaccination during pregnancy $(\mathrm{p}=0.814)$. The mothers of $75.5 \%$ of the newborns who received intensive care service and $74.5 \%$ of those who did not use the service had been vaccinated during their pregnancy. No statistically significant correlation was found between neonatal mortality and vaccination during pregnancy $(\mathrm{p}=0.765)$. The mothers of $78.6 \%$ of those experienced neonatal mortality and $75.1 \%$ of those who did not experience neonatal mortality had been vaccinated (Table 2 ). 
Table 1. Comparison of descriptive characteristics of maternal groups.

\begin{tabular}{|c|c|c|c|}
\hline & $\begin{array}{c}\text { TDV-positive at } \\
\text { pregnancy }(n=327)\end{array}$ & $\begin{array}{c}\text { TDV-negative at } \\
\text { pregnancy }(n=108)\end{array}$ & $p$ value \\
\hline Age $^{*}$ & $29(17-46)$ & $29.5(16-43)$ & 0.751 \\
\hline BMI* $^{*}$ & $24.6(16-43)$ & $25.5(15-45)$ & 0.475 \\
\hline \multicolumn{4}{|l|}{$\mathrm{BMI}^{* *}$} \\
\hline$<25$ & $176(77.8 \%)$ & $51(22.2 \%)$ & \multirow{3}{*}{0.338} \\
\hline $25-30$ & $91(75 \%)$ & $31(25 \%)$ & \\
\hline$>30$ & $60(69.8 \%)$ & $26(30.2 \%)$ & \\
\hline \multicolumn{4}{|c|}{ Place of residence ${ }^{* *}$} \\
\hline Urban & $152(82.2 \%)$ & $33(17.8 \%)$ & \multirow{2}{*}{0.004} \\
\hline Rural & $175(70 \%)$ & $75(30 \%)$ & \\
\hline Nulliparae $e^{* *}$ & $161(82.1 \%)$ & $35(17.9 \%)$ & 0.002 \\
\hline Multiparae $* *$ & $166(69.5 \%)$ & $73(30.5 \%)$ & \\
\hline Smokers ${ }^{* *}$ & $36(73.5 \%)$ & $13(26.5 \%)$ & 0.907 \\
\hline Nonsmokers ${ }^{* *}$ & $291(75.4 \%)$ & 95 (24.6\%) & \\
\hline
\end{tabular}

The statistical significance level was accepted as $\mathrm{p}<0.05 .{ }^{*}$ Median (min-max), ${ }^{* *}$ Frequency (percentage).

Table 2. Maternal-neonatal outcomes.

\begin{tabular}{|c|c|c|c|}
\hline & $\begin{array}{c}\text { TDV-Positive in } \\
\text { Pregnancy }(\mathrm{n}=327)\end{array}$ & $\begin{array}{l}\text { TDV-Negative in } \\
\text { Pregnancy }(\mathrm{n}=108)\end{array}$ & $\mathrm{p}$ value \\
\hline Birth Week ${ }^{*}$ & $38(24-41)$ & $37.6(25-41)$ & 0.992 \\
\hline Birth Weight* & $3070(430-4510)$ & $3067.5(620-4335)$ & 0.995 \\
\hline Normal Newborn ${ }^{* *}$ & $189(57.8 \%)$ & $64(59.3 \%)$ & 0.789 \\
\hline Preeclampsia ${ }^{* *}$ & $48(14.7 \%)$ & $13(12 \%)$ & 0.474 \\
\hline Prematurity ${ }^{\star *}$ & $79(24.2 \%)$ & $24(22.2 \%)$ & 0.677 \\
\hline $\mathrm{SGA}^{\star *}$ & $36(11 \%)$ & $12(11.1 \%)$ & 0.977 \\
\hline $\begin{array}{l}\text { Diabetic Mother's } \\
\text { Infant }\end{array}$ & $12(3.7 \%)$ & $2(1.9 \%)$ & 0.533 \\
\hline Preterm Labor $* *$ & $14(4.3 \%)$ & $5(4.6 \%)$ & 0.793 \\
\hline $\begin{array}{l}\text { Early Membrane } \\
\text { Rupture** }\end{array}$ & $10(\% 3.1)$ & $3(\% 2.8)$ & 1.0 \\
\hline $\begin{array}{c}\text { Temporary Neonatal } \\
\text { Tachypnea }^{* *}\end{array}$ & $16(4.9 \%)$ & $7(6.5 \%)$ & 0.619 \\
\hline \multicolumn{4}{|l|}{$\begin{array}{c}\text { Intensive Care Unit } \\
\text { Service }\end{array}$} \\
\hline Not received ${ }^{* *}$ & $114(74.5 \%)$ & $39(25.5 \%)$ & 0.814 \\
\hline \multicolumn{4}{|l|}{ Neonatal Death } \\
\hline $\begin{array}{l}\mathrm{Yes}^{\star *} \\
\mathrm{No}^{* *}\end{array}$ & $\begin{array}{l}11(78.6 \%) \\
316(75.1 \%)\end{array}$ & $105(24.9 \%)$ & 0.765 \\
\hline
\end{tabular}

The statistical significance level was accepted as $\mathrm{p}<0.05 .{ }^{*}$ Median (min-max), ${ }^{* *}$ Frequency (percentage). 


\section{Discussion}

Vaccines are antigenic preparations that generally contain weakened or killed infectious agents and toxins to stimulate an immune response. Vaccination against diseases has been a matter of dispute for years in the world of science. Causing an increase in inflammatory cytokines (IL-6, TNF- $\alpha$ ) and a decrease in anti-inflammatory cytokines (IL-10), antigenic agents entering the body may lead to a changed immune response, increased anti-angiogenic response, abnormal trophoblast invasion and increased oxidative stress, resulting in preeclampsia. Maternal infections and particularly urinary infections, as well as periodontal disease, have been reported to increase the risk of preeclampsia [6]. For this reason, pathogens may serve as major activators of the inflammatory process in preeclampsia. Through the same mechanisms, tetanus vaccine can be thought of having an impact on the etiopathogenesis of preeclampsia. More than one factor is agreed to play a role in the development of preeclampsia. The factors considered important today include placenta implantation involving abnormal trophoblastic invasion to uterine vessels, unbalanced immunological tolerance between maternal-paternal (placental) and fetal tissues, failure in maternal adaptation to the cardiovascular or inflammatory changes of a normal pregnancy, and genetic factors [7]. The immunological and physiological changes occurring in pregnancy may alter the vulnerability of the mother and the fetus to some infectious diseases, increasing the risk of producing more serious outcomes [8]. In October 2011, the Advisory Committee on Immunization Practices (ACIP) conceived that the potential of transplacental delivery of maternal antibodies to protect the infant against pertussis was superior to postpartum management and recommended to administer diphtheria, tetanus and acellular pertussis vaccines to unvaccinated women towards the end of their second trimester or in their third trimester of pregnancy. This was followed by ACOG's quick acceptance of the ACIP recommendations in March 2012. ACIP resolved that all pregnant women should be vaccinated with TDV during each of their pregnancies and preferably between gestational weeks 27 and 36 regardless of their vaccination certificates [9]. The World Health Organization has defined pregnancy as a special indication for vaccination against influenza and tetanus [10] [11]. The most suitable time for diphtheria, tetanus and acellular pertussis vaccines is considered to be within the third trimester or at least 2 weeks before birth to allow adequate maternal antibody response [12]. The safety of tetanus toxoid vaccine as evidenced in the last 40 years, as well as the $90 \%$ decrease in tetanus-related neonatal mortality, has promoted ideas supporting the use of vaccines during pregnancy [13]. To eradicate maternal and neonatal tetanus, tetanus toxoid (TT) and TDV vaccines are recommended during pregnancy especially in developing countries. In a study comparing TDV and TT in pregnant women, no difference was found in the local (redness and swelling in the injection site) or systemic (fever, weakness, body pain, headache) side effects [14]. Jamie L. Morgan et al. have evaluated in a retrospective study the gestational 
outcomes of women who had TDV after their $32^{\text {nd }}$ gestational week. In their study including a total of 7378 women, 7152 vaccinated and 226 unvaccinated, they did not find any differences between the groups in stillbirths, major malformations, chorioamnionitis, $5^{\text {th }}$ minute Apgar score or cord blood $\mathrm{pH}$, nor in neonatal complications including newborn's ventilation need, sepsis, intraventricular bleeding and neonatal mortality; they were similar to each other. However, preterm birth rates, SGA incidences, and newborn's hospital stay were significantly more in the unvaccinated group. The authors argued that the increase in the preterm births after gestational week 32 in women who refused vaccination could relate to the fact that such women had less opportunity for an antepartum vaccine administration. A comparison of the women who had at least 2 TDVs $(n=1229)$ and those who had only 1 dose $(n=4159)$ in the past 5 years showed that there was a small but significant increase in the mean birth weight in favor of the women who had more than one TDV, but there was no difference in neonatal outcomes [15]. Elyse Olshen Kharbanda et al. retrospectively reviewed 53,885 women who received TDV in some period of their pregnancy and 109,255 women who did not receive TDV in their pregnancy. Most of the vaccinations $(75 \%)$ were in the third trimester. No serious medical conditions were reported 0 - 3 days after the vaccination other than the occurrence of an allergic reaction, fever, and weakness, seizure, mood change, and local lesions in 43 $(8.1 / 10,000)$ of the 53,885 women who had TDV anytime during their pregnancy. For the women who were vaccinated after their $20^{\text {th }}$ gestational week, there was no increased risk of gestational diabetes, thrombocytopenia, venous thromboembolism or cardiac events (myocarditis, pericarditis, cardiomyopathy or heart failure) within 42 days following the vaccination [16]. In a retrospective study evaluating 1759 women who gave birth to a single child between 2012 and 2014, Abbey B. Berenson et al. compared women who had TDV and those who did not with respect to chorioamnionitis, postpartum endometritis, preterm birth, early membrane rupture, birth induction and delivery type, low birth weight, very low birth weight, TT, $5^{\text {th }}$ minute Apgar score, birth defects, and admittance to neonatal intensive unit. Among the infants admitted to neonatal intensive care units, those in the vaccinated group had a shorter stay in the neonatal intensive care unit and the infants in this group were admitted less often due to preterm birth. However, none of the neonatal and maternal outcomes differed with respect to the TDV status. They found small differences with respect to delivery type; vaccinated mothers had fewer deliveries with cesarean section than those unvaccinated [17]. Elyse O. Kharbanda et al. reviewed 123,494 women who had single pregnancies resulting in live births between 1 January 2010 and 15 November 2012 in their retrospective, observational cohort study using the Vaccine Safety Datalink (VSD) website and the Kaiser Permanente Northern California and Kaiser Permanente Southern California databases for the current analyses. Of these women, 26,229 had TDV during their pregnancy and 97,265 did not. TDV was administered in the second and third trimesters in 
$92 \%$ of the 26,229 women and it was administered to $46 \%$ of the 97,265 women before their pregnancies. They investigated chorioamnionitis, hypertensive disorders, SGA and prematurity in that study. SGA, prematurity and hypertensive disorders did not have any vaccine-related risks, but there was small but statistically significant increase in the risk of chorioamnionitis. While $6.1 \%$ of the women who had TDV anytime during their pregnancy were diagnosed with chorioamnionitis, it was seen in $5 \%$ of the women who were not exposed to vaccination. A hypertensive disorder developed before the gestational week 20 in $8.2 \%$ of the women vaccinated with TDV and $8 \%$ of those unvaccinated [18]. According to the prospective, controlled cohort study made by Kirsten Maertensa et al., 57 pregnant women were vaccinated with TDV at their gestational week 28.6 on the average. There were 42 unvaccinated pregnant women in their control group. Mild symptoms (hardness at the injection site, vaginal discharge, reflux, fever < $38.5 \mathrm{C}$, broad leg swelling, redness in abdomen and arms) were seen in 46 out of 57 women in the vaccine group, but they healed in 72 hours. Adverse clinical conditions that, according to the opinion of the investigator, are not associated with the vaccine and not different from those seen in the general population (preeclampsia, preterm labor, hypertension, oligohydramnios and placenta previa) were reported in the vaccine group [19]. In a study made on the database of the Vaccine Adverse Event Reporting System (VAERS) between 2005 and 2010, it was reported that TDV vaccination during pregnancy was not associated with any maternal or neonatal outcomes [9]-[20]. The randomized, controlled study of Dabrera G. et al. exploring maternal and neonatal outcomes in 48 pregnant women reported that there was no increased risk of adverse gestational or neonatal events that could be attributed to vaccination [21]. Katherine Donegan et al. who reviewed the data in the UK Clinical Practice Research Datalink and assessed 20,074 pregnant women did not find any evidence of an increased risk of stillbirth, maternal or neonatal mortality, preeclampsia or eclampsia, bleeding, fetal distress, uterine rupture, placenta or vasa previa, delivery with cesarean section, low birth weight or neonatal kidney failure [22]. We also made a comparison between the groups in our study on patients with similar characteristics with respect to gestational week, birth weight, newborn health condition, small for gestational age (SGA), early membrane rupture, preterm labor, diabetic mother's baby, prematurity, temporary neonatal tachypnea, newborn's need for intensive care and neonatal mortality and we obtained similar results. The limitations of the study were that the study was retrospective, that the data contained only the results of one region, and that we did not have any data to evaluate the long-term neonatal results between the groups.

\section{Conclusion}

No significant correlation was found in this study between TDV administered during pregnancy and adverse gestational outcomes. Vaccination during pregnancy was found correlated with the place of residence. While $82.2 \%$ of those 
living in urban areas had the vaccination, only $70 \%$ of those living in rural areas were vaccinated. The lower rate of vaccination in rural areas may be associated with the restrictions in having access to health services in the periphery. A statistically significant correlation was found also between nulliparity and vaccination status. While the rate of vaccination was $69.5 \%$ in multiparae, it was $82.1 \%$ in nulliparae. This may be associated with the fact that women who had their first pregnancy attend health checks more regularly. There are very few studies investigating the relationship between diphtheria-tetanus vaccination during pregnancy and development of preeclampsia. Our study demonstrated the fact that women who had TDV during their pregnancy do not exhibit a significant difference in maternal and neonatal outcomes as compared to those who were unvaccinated.

\section{Conflicts of Interest}

The authors declare no conflicts of interest regarding the publication of this paper.

\section{References}

[1] American College of Obstetricians and Gynecologists (2013) Hypertension in Pregnancy. Report of the American College of Obstetricians and Gynecologists' Task Force on Hypertension in Pregnancy. Obstetrics and Gynecology, 122, 1122.

[2] Sibai, BM. and Stella, C.L. (2009) Diagnosis and Management of Atypical Preeclampsia-Eclampsia. American Journal of Obstetrics and Gynecology, 200, 481-e1. https://doi.org/10.1016/j.ajog.2008.07.048

[3] Elhawary, T.M., El-Bendary, AS. and Demerdash, H. (2012) Maternal Serum Endoglin as an Early Marker of Pre-Eclampsia in High-Risk Patients. International Journal of Women's Health, 4, 521. https://doi.org/10.2147/IJWH.S35318

[4] Gautret, P. and Wilder-Smith, A. (2010) Vaccination against Tetanus, Diphtheria, Pertussis, and Poliomyelitis in Adult Travelers. Travel Medicine and Infectious Disease, 8, 155-160. https://doi.org/10.1016/j.tmaid.2010.02.007

[5] Kliegman, R.M., Stanton, B.F., Schor, N.F., St. Geme III, J.W. and Behrman, R.E. (2015) Nelson Pediatric. Saunders, Phialdelphia, 991-994.

[6] Agudelo, C., Villar, J. and Lindheimer, M. (2008) Maternal Infection and Risk of Preeclampsia: Systematic Review and Metaanalysis. American Journal of Obstetrics \& Gynecology, 198, 7. https://doi.org/10.1016/j.ajog.2007.07.040

[7] Cunningham, F.G., Leveno, K.J., Bloom, S.L., Spong, C.Y., Dashe, J.S., Hoffman, B.L., Casey, B.M. and Sheffield, J.S. (2016) Williams Obstetrics. 24th Edition, McGraw-Hill Professional Publishing, New York, 728-779.

[8] Jamieson, D.J., Theiler, R.N. and Rasmussen, S.A. (2006) Emerging Infections and Pregnancy. Emerging Infectious Diseases Journal, 12, 1638-1643.

https://doi.org/10.3201/eid1211.060152

[9] Centers for Disease Control and Prevention (CDC) (2013) Updated Recommendations for Use of Tetanus Toxoid, Reduced Diphtheria Toxoid, and Acellular Pertussis Vaccine (Tdap) in Pregnant Women: Advisory Committee on Immunization Practices (ACIP), 2012. Morbidity and Mortality Weekly Report, 62, 131-135.

[10] WHO (2012) Vaccines against Influenza WHO Position Paper. The Weekly Epide- 
miological Record, 87, 461-476.

[11] WHO (2006) Tetanus Vaccine. The Weekly Epidemiological Record, 81, 198-208.

[12] Swamy, G.K. and Heine, R.P. (2015) Vaccinations for Pregnant Women. Obstetrics \& Gynecology, 125, 212-226. https://doi.org/10.1097/AOG.0000000000000581

[13] Elimination of Maternal and Neonatal Tetanus, Health, UNICEF. https://www.unicef.org/health/index_43509.html

[14] Salama, M.M., Hady, O.A., Ashour, W., Mostafa, A., El Alkamy, S., El Sayed, N., et al. (2009) A Randomized Controlled Trial Administration of Tetanus Toxoid (TT) versus Tetanus and Reduced Diphtheria (Td) in Pregnant Women. Journal of Clinical Immunology, 29, 524-531. https://doi.org/10.1007/s10875-009-9289-5

[15] Morgan, J.L., Baggari, S.R., McIntire, D.D. and Sheffield, J.S. (2015) Pregnancy Outcomes after Antepartum Tetanus, Diphtheria, and Acellular Pertussis Vaccination. Obstetrics \& Gynecology, 125, 1433-1438. https://doi.org/10.1097/AOG.0000000000000862

[16] Kharbanda, E.O., Vazquez-Benitez, G., Lipkind, H.S., Klein, N.P., Cheetham, T.C., Nalewaye, A.L., Lee, G.M., Hambidgeg, S., Jackson, M.L., Omeri, S.B., McCarthyj, N. and Nordina, J.D. (2016) Maternal Tdap Vaccination: Coverage and Acute Safety Outcomes in the Vaccine Safety Datalink, 2007-2013. Vaccine, 34, 968-973.

[17] Berenson, A.B., Hirth, J.M., Rahman, M., Laz, T.H., Rupp, R.E. and Sarpong, K.O. (2016) Maternal and İnfant Outcomes among Women Vaccinated against Pertussis during Pregnancy. Human Vaccines \& Immunotherapeutics, 12, 1965-1971. https://doi.org/10.1080/21645515.2016.1157241

[18] Kharbanda, E.O., Vazquez-Benitez, G., Lipkind, H.S., Klein, N.P., Cheetham, T.C., Naleway, A., McCarthy, N.L., et al. (2014) Evaluation of the Association of Maternal Pertussis Vaccination with Obstetric Events and Birth Outcomes. Jama, 312, 1897-1904. https://doi.org/10.1001/jama.2014.14825

[19] Maertens, K., Caboré, R.N., Huygen, K., Hens, N., Van Damme, P. and Leuridan, E. (2016) Pertussis Vaccination during Pregnancy in Belgium: Results of a Prospective Controlled Cohort Study. Vaccine, 34, 142-150. https://doi.org/10.1016/j.vaccine.2015.10.100

[20] Zheteyeva, Y.A., Moro, P.L., Tepper, N.K., Rasmussen, S.A., Barash, F.E., Revzina, N.V., et al. (2012) Adverse Event Reports after Tetanustoxoid, Reduced Diphtheria Toxoid, and Acellular Pertussis Vaccines in Pregnant Women. American Journal of Obstetrics \& Gynecology, 207, 59.e1-7. https://doi.org/10.1016/j.ajog.2012.05.006

[21] Dabrera, G., Amirthalingam, G., Andrews, N., Campbell, H., Ribeiro, S., Kara, E., et al. (2015) A Case-Control Study to Estimate the Effectiveness of Maternal Pertussis Vaccination in Protecting Newborn İnfants in England and Whales, 2012-2013. Clinical Infectious Diseases, 60, 333-337. https://doi.org/10.1093/cid/ciu821

[22] Donegan, K., King, B. and Bryan, P. (2014) Safety of Pertussis Vaccination in Pregnant Women in UK: Observational Study. BMJ, 349, g4219.

https://doi.org/10.1136/bmj.g4219 